\title{
Mitochondrial DNA Variants in Autism Spectrum Disorder: A Systematic Review
}

\section{Liao Xiaoli ( $1145869595 @ q q . c o m)$}

Zhongnan University of Economics and Law

Li Yamin

Central South University

\section{Research}

Keywords: Autism Spectrum Disorder, Mitochondrial, mtDNA, mtDNA variants

Posted Date: December 21st, 2020

DOI: https://doi.org/10.21203/rs.3.rs-131427/v1

License: (c) (i) This work is licensed under a Creative Commons Attribution 4.0 International License. Read Full License 


\section{Abstract}

Objectives The present review aimed to systematically characterize existing literature regarding the involvement of mtDNA variants in the pathophysiology of ASD.

Methods A systematic search of publications in three online databases plus a manual search of reference lists of included articles was performed to collate potentially eligible literature. The study selection and data extraction were conducted by two independent authors, and the discrepancies in each step were settled through discussions.

Results From 1874 resulting searched articles, 28 studies remained for this review.

These included studies involving 3 studies reported ancient haplogroup variants, 16 studies reported point mutations, 7 studies reported copy number variants, and 8 studies reported deletions, with point mutation being the most common type of disease-related variants.

Conclusions Findings generated from this review support mtDNA variants as important contributory components in susceptibility to ASD, since most variants have yet been mapped to specific loci, and the exact pathogenic mechanism remains to be determined.

\section{Background}

\section{Autism spectrum disorder}

Autism spectrum disorder (ASD) is a neurodevelopmental disorder characterized by the impairment of socio-communicative skills and the presence of stereotyped behavior patterns (Lord et al., 2000). The ASD incidence has markedly increased 10 to 17 percent per year during the last two decades (Blumberg et al., 2013; Christensen et al., 2016), with the most current estimates being about $1 / 54$ children in the Unite States (US) (Maenner et al., 2020). The considerable increase in ASD prevalence has stimulated worldwide research interests into the etiology of this disorder, which mainly points to environmental factors acting on genetic predispositions involving both nuclear DNA (nDNA) and mitochondrial DNA (mtDNA) (Dhillon et al., 2011; Persico \& Napolioni, 2013). Despite considerable efforts in the understanding of its etiological mechanism and biological basis, no reliable biomarker or distinct pathogenesis has yet been established for ASD (de la Torre-Ubieta et al., 2016; Frye \& Rossignol, 2011).

Since Lombard (1998) first proposed that ASD may be a disorder of impaired mitochondrial function in 1998 (Lombard, 1998), growing number of studies points to a potential involvement of mitochondrial physiology in the pathophysiological pathway of ASD, with evidence from genetic, post-mortem, neuroimaging and biomarker studies in both ASD subjects and animal models. Converging lines of evidence indicates that both oxidative stress and abnormal energy metabolism may contribute to the etiology of ASD, consistent with some level of mitochondrial dysfunction (Clark-Taylor \& Clark-Taylor 2004; Oliveira et al., 2005; Rossignol et al., 2007), although this remains somewhat controversial (Lerman-Sagie et al., 
2004). Although increasing evidence supports the involvement of mitochondrial dysfunction in the pathophysiology of ASD, the underlying biological basis of this connection remains relatively unexplored (Legido et al., 2013). The genetic complexity of ASD together with the association of ASD with mitochondrial dysfunction provides a strong rationale for investigating the role of mtDNA in the etiology of the ASD (Crimi et al., 2004; Dhillon et al., 2011; Zsurka \& Kunz, 2015). While the nuclear genome has been the focus of countless studies over the past decades, the potential role of mtDNA variants in the etiology of ASD remains relatively unexplored and warrants thorough investigation.

\section{Mitochondria}

Mitochondria are double membrane-bound organelles responsible for energy metabolism in eukaryotic cells, which controlled by both nuclear DNA (nDNA) and mitochondrial DNA (mtDNA). The number of mitochondria in each cell and the amount of mtDNA in each mitochondria vary according to the bioenergetic needs of particular cell and the levels of oxidative cellular stress, having the brain as the most energetically demanding tissue in human body (Valenti et al., 2014).

The human mtDNA is a double-stranded supercoiled ring molecule, which exclusively inherits from the matrilineal line. The human mtDNA contains 15,000-17,000 base pairs which codes 37 genes expressing 13 polypeptides involved in OXPHOS, 22 tRNAs as well as 2 rRNAs. As lack of protective histones, less efficient system for DNA repair along with high level of reactive oxygen species (ROS) generation in this organelle (Lin \& Beal, 2006), mtDNA presents a very high variants rate in comparison to nDNA (Napoli et al., 2013). The rate of mtDNA variants depends mainly on the level of oxidative stress and the fidelity of the mtDNA polymerase (Li et al., 2019). Clinically relevant mtDNA variants fall into three classes, including recent deleterious mutations, ancient adaptive variants, and de novo mutations or somatic mutations (Wallace, 2015).

The brain has the highest energy requirement, accounting for $2-3 \%$ of human body weight but using $20 \%$ of the total mitochondrial energy, making it the most sensitive organ to mtDNA variants (Wallace, 2013). Since the first report of an inherited mtDNA disease variant three decades ago (Wallace et al., 1988), hundreds of clinically relevant mtDNA variants have been identified parallel with the breakthrough of sequencing approaches and cell biology technologies (Schon et al., 2012). Genetic variants in mtDNA can give rise to mitochondrial dysfunction, resulting in abnormal cellular energy production, impaired intracellular calcium buffering and accumulated reactive oxygen species,with deleterious consequences for normal physiological function, contributing to the development of a diverse set of disorders, ranging from neurodegenerative diseases, to neuropsychiatric disorders, and to neurodevelopmental syndromes such as ASD (Cruz et al., 2019; Hoekstra et al., 2016; Li et al., 2016; Patowary et al., 2017).

Previous studies of ASD susceptibility genes mainly focused on nDNA, which may neglect a fact that the mtDNA is central to the study of evolutionary genetics, and the human mtDNA is one of the first completely sequenced mammalian genomes. Increasing evidence indicates that mtDNA variants may affect the energy supply and key processes of the developing brain, triggering a cascade of structural and 
functional changes, thereby contributing to the etiology of ASD (Chauhan et al., 2012; Gargus \& Imtiaz, 2008; Palmieri \& Persico, 2010; Rossignol \& Frye, 2012).

\section{Objective}

Although increasing reviews have described the potential role of mitochondrial dysfunction in general, and of mtDNA variants in particular, in a variety of neurological disorders (Greaves et al., 2012; Koopman et al., 2012; Schon \& Przedborski, 2011; Ylikallio \& Suomalainen, 2012), there has been no systematic review specifically appraising the role of mtDNA variants in the context of ASD. The present review aims to systematically summarize existing evidence regarding the involvement of mtDNA variants in the etiology of ASD.

\section{Methods}

\section{Search strategy}

A electronic search was performed in three databases, PubMed, Embase and Web of science, using the following keywords (Autism Spectrum Disorder OR Autistic disorder OR ASD OR autism OR autistic OR Asperger Syndrome OR Pervasive Developmental Disorder) AND (mitochondria OR mitochondrial OR mitochondrion) AND (genetic OR RNA OR DNA OR genome OR transcript OR gene).

No restrictions were placed on the study location or the publication year. All authors achieved a consensus on the search strategies. References cited by all eligible citations and previous reviews were manually searched for additional relevant studies that might have been omitted.

\section{Selection Criteria}

The following inclusion criteria were required: published in English language; focused on subjects with ASD; presented genetic variants in mtDNA. The following exclusion criteria were also required: studies with non-human samples; reported theoretical outcomes (eg. reviews, editorials, letters and commentaries).

\section{Results}

\section{Literature Search}

Figure 1 summarized the results of the search strategy and study selection processes. A total of 1874 records were generated from the initial search of these three databases. After eliminating 215 duplicates, 1659 articles were considered potentially relevant for subsequent analysis. The screening of titles plus the analysis of abstracts ruled out 1428 articles that lacked relevance, leaving 231 citations for further screening. The analysis of full texts combine with the application of inclusion/exclusion criteria excluded 203 articles that failed to meet the eligible criteria, yielding 28 articles as the source documents for this review. 


\section{Study Characteristics}

Table 1 summarized the main characteristics and findings of these included studies.

These included articles used various research methods, involving case-control $(n=14)$, case-series $(n=6)$, case report $(n=5)$, cohort analysis $(n=2)$ and cross sectional $(n=1)$ designs. The 14 case-control studies comprising a total of 7,414 subjects, including 3060 ASD and 4354 matched controls. The 6 case-series studies containing a whole of 126 subjects, sample sizes ranged from 10 to 31 . These studies included subjects spanned the entire childhood population, from young children to adolescents. The samples used in these studies included many different types of tissues, including blood, muscle, skin, oral mucosa, postmortem brain, with peripheral blood sample being the most common cell model for studies.

\section{Haplogroup}

The haplogroup is defined as the individual group of mtDNA, characterized by the existence of a specific set of mtDNA polymorphisms that have accumulated continuously along the radiating maternal lineages. The search yielded 3 studies reported the potential involvement of ancient haplogroup-associated mtDNA variants in the development of ASD, although controversy has existed.

The involvement of mitochondrial haplogroup in pathogenesis of ASD has been supported by result from Chalkia et al.(2017), who appraised the potential impact of ancient mtDNA polymorphisms in the risk of developing ASD in a cohort study. The result showed that European haplogroups I, J, K, O-X, T, and U were associated with increased ASD risks, as were Asian and Native American haplogroups $A$ and $M$. No compelling evidence of an association of any mitochondrial haplogroup to the risk of developing ASD has been found in more comprehensive case-control studies. In order to explore the association between mitochondrial haplogroup and ASD, Kent et al.(2008) charecterised the mtDNA haplogroup in a cohort of 162ASD probands and compared to two sets of population controls, reported no compelling evidence of an association between any mitochondrial haplogroup and ASD risk. Hadjixenofontos et al.(2013) examined the role of mtDNA variants in the risk of developing ASD through a multi-phase approach, found no evidence of the involvement of European haplogroups in genetic etiology of ASDs.

\section{Single Nucleotide Variants (snvs)}

SNPs exist in all protein coding mtDNA molecules, which appear to be the most frequent type of diseased-related mtDNA mutation. The search yielded a total of 16 studies reported a pathological role of mtDNA point mutations in the development of ASD, but still somewhat inconsistent.

A total of 13 studies supported a role of mtDNA point mutation in the the etiology of ASD. Early cases reported by Graf et al. (2000), who identified a variant $\mathrm{m} .8363 \mathrm{G}>\mathrm{A}$ in the MT-TK gene in a 3 year old boy with ASD, found a $60 \%$ mutation rate in muscle tissue and $61 \%$ in blood. Pons et al.(2004) followed five unrelated children with ASD, found that children with ASD with or without additional neurologic features can be early presentations of the $m .3243 A>G$ mutation in the tRNA ${ }^{\text {Leu(UUR) }}$ gene. A report from Frye 
(2012) characterized mtDNA mutation in two ASD boys, identified a novel mutation located in the MTCYB gene in both of the boys. Additional evidence from Connolly et al.(2010), who reported the mtDNA mutation in a Canada family, identified a variant m.3460G > A in the MT-ND1 gene as a pathogenic mutation associated with ASD. Weissman et al.(2008) reviewed the medical records of 25 ASD subjects with evidence of a definite or probable mitochondrial disorder. Results from genomic analysis showed that two subjects presented variant m.3397A $>\mathrm{G}$ in the MT-ND1 gene and variant $\mathrm{m} .4295 \mathrm{~A}>\mathrm{G}$ in the in the tRNA lleu gene, one subjects harbored variant m.11984T > $C$ in the MT-ND4 gene. Piryaei et al.(2012) examined the role of mtDNA variants in the pathogenesis of ASD in a sample of 24 ASD subjects, identified 3 new mtDNA mutations in a portion of the 24 subjects, including variant $\mathrm{m} .8472 \mathrm{C}>\mathrm{A}$ located in the MT-ATP8 gene and variant $\mathrm{m} .8697 \mathrm{G}>\mathrm{A}$ located in the MT-ATP6 gene in 3 of these subjects, as well as variant $\mathrm{m} .8697 \mathrm{G}>\mathrm{A}$ located in the MT-ATP6 gene in 5 of these subjects. The study of AvdjievaTzavella et al.(2012) analyzed mtDNA variants in 21 children with ASD, found potentially disease-causing mtDNA variants in two children, with the non-synonymous mutation m.6852G > A (Gly317Ser) located in the MT-CO1 gene and the non-synonymous variant m.8033A > G located in the MT-CO2 gene. Napoli et al. (2013) evaluated the quality of mtDNA (sequence variants) in peripheral blood of children with ASD, identified a higher frequency of GC > AT transitions and G,C,T > A transitions in ASD subjects as compared to controls. A recent study provided evidence of an association between ASD and increased inherited mutations from the mother by Wang et al.(2016), who investigated the genetic relationship between biological siblings in a sample of 903 simplex families, reported that both non-synonymous mutations and predicted pathogenic synonymous mutations conferred an increased risk for ASD, with higher frequency of these mutations in individuals with lower IQ and/or deficit in social behavior. Djordjevic et al. (2016) described novel mitochondrial mutations associated with a complex mitochondrial encephalopathy, identified a mutation m.7495A > G located in the MT-TS1 gene in a four year old female with ASD characteristics who presented $83 \%$ of heteroplasmy. Patowary et al. (2017) analyzed mtDNA in 10 subjects with familial ASD by employing Next generation sequencing approaches. The result identified two variants in MT-ND5 gene within a single family and a variant in MT-ATP6 gene in a second family. The study of Valiente-Pallejà et al. (2018) examined the presence of mtDNA alterations in a cohort of 122 ASD subjects and matched controls, found that $26.6 \%$ of ASD subjects carried at least one putative pathogenic mtDNA mutation. Akouchekian et al.(2019) examined the role of mtDNA variants in the development of ASD by focusing on COX1-3 genes. The result identified 15 unique variants in COX1-3 genes, with the most common variant being the amino acid change $A->A$.

The evidence was not entirely concordant, other studies failed to identify an association between mtDNA point mutations and ASD. Oliveira et al. (2005) reported the first population based study to identify mtDNA variants in ASD, yielding no mtDNA variants in ASD subjects. Álvarez-Iglesias et al.(2011) characterised mtDNA variants in a cohort of 148 ASD subjects, reported no statistical support for an association between mtDNA mutations and ASD. Filiano et al.(2002) illustrated the importance of the mitochondria in children with features of ASD, identified no mtDNA point mutations in these children despite the presence of a $7.4 \mathrm{~kb}$ mtDNA mutation in one child.

\section{Copy Number Variants (cnvs)}


CNVs has been proved to be the most prevalent type of structural variations in human genome (lafrate et al., 2004; Redon et al., 2006), and CNVs of mtAND appeared to be one of the most common biomarkers of mitochondrial dysfunction. The copy number of mtDNA varies according to the energy requirements of cells and levels of oxidative cellular stress (Gu et al., 2013), which has been associated with mitochondrial dysfunction and increased oxidative stress (Malik \& Czajka 2013). An involvement of mtDNA variants in the development of ASD has been supported by findings of altered mtDNA copy number in ASD subjects, although this remained somewhat controversial.

The search yielded 5 studies that reported an increased mtDNA copy number in ASD subjects as compared to controls, which could account for a compensatory mechanism to strengthen the different mitochondrial functions that govern cellular bioenergetics. Carrasco et al.(2019) characterised the influence of mitochondrial physiology on the development of ASD, found a significant increased mtDNA contents in the oral mucosa of ASD subjects as compared to matched controls. Gu et al.(2013) compared the copy number and deletions of Cyt B, ND1 and ND4 genes in the frontal cortex of ASD subjects and age-matched controls, reported an increased copy numbers of these three mitochondrial genes, as well as deletion of ND4 and Cyt B in the frontal cortex of ASD subjects relative to controls. Yoo et al.(2017) examined the association between mitochondrial dysfunction and ASD phenotypes, found a significant higher level of mtDNA copies in the peripheral blood of probands with ASD to that of their unaffected sib pairs, and it was correlated to a subtype of phenotype presenting communication deficits. Chen et al. (2015) examined the mtDNA copy number in peripheral blood cells from ASD subjects and healthy controls, found a significant increased mtDNA copy number of MT-ND1, MT-ND4 and MT-CYB genes in ASD subjects as compared to healthy controls, but it was not related to clinical features of ASD. Wong et al.(2016) quantified the mtDNA copy number in peripheral blood monocytic cells from 66 children with ASD and 46 typically developing children, found a significant higher number of mtDNA copies in children with ASD relative to typically children.

Also one study reported a decreased mtDNA copy number in ASD subjects relative to controls. ValientePallejà et al.(2018) examined the content of mtDNA in a cohort of 122 ASD subjects and matched controls, reported a significant lower number of mtDNA copies in both MT-ND1 and MT-ND4 genes in ASD subjects as compared to healthy controls.

However, this finding could not be reproduced in a following postmortem study of Tang et al.(2013), who characterized mtDNA variants in a larger cohort of postmortem temporal lobe from ASD subjects and healthy controls, reported no significant difference in mtDNA copy number between ASD subjecst and controls.

\section{Deletions}

Deletions, a special type of copy number variants, responsible for the absence of a part of the mitochondrial genome. The literature search generated a total of 8 studies reported the potential pathogenicity of mtDNA deletions in the development of ASD, yielding inconsistent results. 
The saerch generated 2 studies regarding the association between mtDNA deletions and ASD only reported the deleted gene in ASD subjects without specifying mtDNA coordinates. Napoli et al.(2013) reported the involvement of mtDNA deletions in the development of ASD, found a significant higher frequency of mtDNA deletions at the segment of MT-CYB and MT-ND4 in ASD subjects relative to controls. Giulivi et al.(2010) evaluated mtDNA abnormalities in a well-defined population of children with ASD compared to healthy controls, found deletions at MT-CYB in 2 cases, along with over-replication in 5 cases.

The search yielded 6 studies that linked mtDNA deletions to ASD only presented the length of mtDNA deletions in ASD subjects without describing the exactly genomic location. Wong et al.(2016) evaluated the role of mtDNA deletions in the development of ASD, found a significant higher incidence of mtDNA deletion in ASD cases as compared to healthy controls. An exploratory study of the correlation between mtDNA changes and ASD carried out by Varga et al.(2018) analyzed common mtDNA variants in 60 ASD patients and 60 healthy controls, found that $16.6 \%$ of ASD subjects presented mtDNA deletions when compared with healthy controls. The work of Shoffner et al.(2010) examined the role of mtDNA variants in the etiology of ASD, found that children with mitochondrial defects had a higher risk in ASD regression, although only one patient presented mtDNA depletion. Filiano et al.(2002) evaluated mtDNA deletions in a group of 12 children who presented clinically with hypotonia, intractable epilepsy, autism, and developmental delay (HEADD syndrome), identified increased levels of large-scale mtDNA deletions in five of these cases, three patients exhibited a $7.4 \mathrm{~kb}$ deletion whereas the remaining two patients harbored a 3.3 to $7.4 \mathrm{~kb}$ deletion. Pons et al.(2004) characterized mtDNA variants in five unrelated children with ASD, found an significant depletion of mtDNA in skeletal muscle in a child with PDD having a history of mtDNA depletion syndrome in an older sister. Increasing body of evidence confirmed the contribution of mtDNA deletions to the pathogenesis of ASD, but also one study rejected this contribution. Tang et al. (2013) examined mitochondrial abnormalities in temporal lobe of ASD brain. Findings from long-range PCR amplification ruled out the presence of large-scale deletions in ASD subjects despite the appearance of oxidative damage.

\section{Discussion}

While the nDNA has been the focus of studies over the past decades in search for ASD susceptibility genes, accumulating evidence emerged to indicate that mtDNA may also account for the genetic etiology of ASD. The present review first provided a brief overview of mitochondria and mtDNA, then performed a systematic review of existing studies concerning the role of mtDNA variants in the etiology of ASD. Findings generated from this review suggested that mtDNA variants can be important contributory components in susceptibility to ASD, since most variants have yet been mapped to specific loci, and the exact pathogenic mechanism remains unclear.

The unique genetics of the mitochondrion can account for many puzzling features in the genetic etiology of ASD. Since mtDNA and nDNA exert a dual genetic control on the biogenesis and maintenance of mitochondrial function, nDNA may also involved in the mitochondrial physiology related to ASD. A series 
of perinatal allergic, genetic, environmental, immune and infectious factors have been identified as contributors to the pathogenesis of ASD through the increase of oxidative stress. The increased oxidative stress can give rise to variants in mtDNA as a result of base modification, mtDNA strand cleavage, and cross-linkage with other molecules, which undergo accumulation and clonal expansion in postmitotic tissues until reaching the phenotypic threshold.

The presence of predisposing mtDNA haplogroups plus the accumulation of mtDNA point mutations, together with additional environmental insults and the interaction with nDNA, would appear to lower mitochondrial function below the minimal bioenergetic threshold for normal brain function, leading to reduced ATP production, increased ROS levels, altered cellular redox state, disturbed calcium homeostasis promoting the development or progression of ASD. This mitochondrial cascade hypothesis may help explain the potential role of mtDNA variants in the pathophysiology of ASD. Furthermore, existing studies indicated that mtDNA variants can change the amino acid sequences of the mtDNAcoded mitochondrial $\mathrm{N}$-formylmethionine-initiated polypeptides, which can be release into the blood stream. The release into the blood stream of these bacterial-like variant polypeptides plus mtDNA can initiate the inflammatory response, which have emerged as crucial pathological components in the pathogenesis of ASD (Krysko et al., 2011; Oka et al., 2012; Zhang et al., 2010).

Having here reviewed evidence on the potential involvement of mtDNA variants in the pathogenesis of ASD, targeting mtDNA variants associated mitochondrial dysfunction appears to be a promising strategy for treating ASD. Increasing studies have begun to examined the potential therapeutic benefits of mitochondrial-targeted molecules in ASD subjects (Delhey et al., 2017; Rose et al., 2018), with some reported improvements in mitochondrial function and clinical phenotypes after the supplementation of quinones (co-enzyme Q and idebenone), vitamin E and C, lipoic acid and ascorbic acid (Pfeffer et al., 2013; Rossignol \& Frye, 2012; Valenti et al., 2014).

\section{Limitation}

Several limitations should be noted before firm conclusions could be drawn from this reviewed. First, although the relevance of mtDNA variants in ASD has been confirmed in this review, definitive conclusions regarding causal inferences can not been drawn due to the inherent limitation of case-control design. Second, findings yielded from this review failed to prove an association between mtDNA variants and clinical phenotypes, since most of these included studies only investigated the presence of mtDNA in ASD. Third, this review focused exclusively on mtDNA variants without taking nDNA into account, which may lead to an incomplete picture of the complex genetic architecture of ASD. Defects in nDNA encoded proteins required for mtDNA maintenance may lead to instability of mtDNA, which can be manifested through the generation of point mutations, multiple deletions, copy number variants,or combinations thereof in mtDNA (Ylikallio \& Suomalainen, 2012). Additional attention should be paid to the heterogeneity among these included studies, including the complex phenotypes of ASD, the frequent presence of comorbid diagnoses, and the limited sample size of each study, which could account for some inconsistent of the results. 


\section{Conclusion}

The present review first briefly outlined the importance of mitochondria and mtDNA in human health and disease, then systematically summarized evidence regarding the role of mtDNA variants in the development of ASD, finally discussed possible explanations for these findings and the limitations of this evidence. Findings generated from this review supports the involvement of mtDNA variants in the underlying pathophysiology of ASD, despite the continuing concern about whether mtDNA variants directly contribute to the pathogenesis of ASD or merely serve as an epiphenomenon of ASD. The increasing understanding of mtDNA variants in the development of ASD will fuel the emergence of clinical diagnostic biomarker and novel therapeutic options acting on the underlying genetic pathogenesis of this disorder.

\section{Abbreviations}




\begin{tabular}{|ll|}
\hline ASD & Autism spectrum disorder \\
\hline ATP & Adenosine triphosphate \\
\hline CNV & Copy number variation \\
\hline ETC & Electron transport chain \\
\hline MD & Mitochondrial dysfunction \\
\hline mtDNA & mitochondrial DNA \\
\hline nDNA & nuclear DNA \\
\hline MT-ATP6 & mitochondrially encoded ATP synthase 6 \\
\hline MT-ATP8 & mitochondrially encoded ATP synthase 8 \\
\hline MT-CO1 & mitochondrially encoded cytochrome c oxidase I \\
\hline MT-CO2 & mitochondrially encoded cytochrome c oxidase II \\
\hline MT-CYB & mitochondrially encoded cytochrome b \\
\hline MT-ND1 & mitochondrially encoded NADH dehydrogenase 1 \\
\hline MT-ND4 & mitochondrially encoded NADH dehydrogenase 4 \\
\hline MT-ND5 & mitochondrially encoded NADH dehydrogenase 5 \\
\hline OXPHOS & oxidative phosphorylation \\
\hline ROS & reactive oxygen species \\
\hline rRNA & ribosomal RNA-encoding genes \\
\hline SNP & single nucleotide polymorphism \\
\hline tRNA & translation RNA-encoding genes \\
\hline
\end{tabular}

\section{Declarations}

\section{Ethics approval and consent to participate}

Not applicable.

\section{Consent for publication}

Not applicable.

\section{Competing interests}

The authors declare that they have no competing interests. 


\section{Funding}

This research was funded by the National Natural Science Foundation of China (NO: 81873806) and the National Natural Science Foundation of Hunan Province (NO: 514 2019JJ40437).

\section{Authors' contributions}

All authors have read and agree to the published version of the manuscript.

\section{Acknowledgements}

Not applicable.

\section{References}

Akouchekian M, Hakim Shooshtari M, Heidary H, Zahedi Abghari F, Moeinian P. The causative variants of amyloidosis in the autism. Int J Neurosci. 2019,129(1):10-15

Álvarez-Iglesias V, et al. (2011) Reassessing the role of mitochondrial DNA mutations in autism spectrum disorder. BMC Med Genet. 2011,12(1): 50

Anitha A, et al. Down regulation of the Expression of Mitochondrial Electron Transport Complex Genes in Autism Brains. Brain Pathol. 2012, 23(3): 294-302

Avdjieva-Tzavella D, et al. Mitochondrial DNA mutations in two Bulgarian children with autistic spectrum disorders. Balk J Med Genet. 2012, 15(2): 47-53

Blumberg SJ, et al. Changes in Prevalence of Parent-Reported Autism Spectrum Disorder in School-Aged US Children: 2007 to 2011-2012. National Center for Health Statistics Reports. 2013,65: 1-11

Carrasco M, Salazar C, Tiznado W, Ruiz LM. Alterations of Mitochondrial Biology in the Oral Mucosa of Chilean Children with Autism Spectrum Disorder (ASD). Cells. 2019, 8(4): 367

Chalkia D, et al. Association between mitochondrial DNA haplogroup variation and autism spectrum disorders. JAMA psychiat. 2017, 74(11): 1161-1168

Chauhan A, Gu F, Chauhan V. Mitochondrial respiratory chain defects in autism and other neurodevelopmental disorders. J Pediatr Biochem.2012, 2: 213-223

Chen S, et al. Elevated mitochondrial DNA copy number in peripheral blood cells is associated with childhood autism. BMC psychiatry. 2015, 15(1):50

Christensen DL, et al. Prevalence and characteristics of Autism Spectrum Disorder among children aged 8 years-Autism and Developmental Disabilities Monitoring Network, 11 Sites, United States, 2012. MMWR Surveill Summ.2016, 65:1-23 
Clark-Taylor T, Clark-Taylor BE. Is autism a disorder of fatty acid metabolism? Possible dysfunction of mitochondrial beta-oxidation by long chain acyl-CoA dehydrogenase. Med Hypotheses.2004, 62: 970-975

Connolly BS, et al. MELAS syndrome, cardiomyopathy, rhabdomyolysis, and autism associated with the A3260G mitochondrial DNA mutation. Biochem Biophys Res Commun.2010, 402(2): 443-447

Crimi $\mathrm{M}$, et al. A missense mutation in the mitochondrial ND5 gene associated with a Leigh-MELAS overlap syndrome. Neurology. 2003, 60:1857-61

Cruz ACP, Ferrasa A, Muotri AR, Herai RH. Frequency and association of mitochondrial genetic variants with neurological disorders. Mitochondrion. 2019, 46: 345-360

de la Torre-Ubieta L, Won H, Stein JL, et al. (2016) Advancing the understanding of autism disease mechanisms through genetics. Nat Med. 22(4):345-361

Delhey LM, et al. The Effect of Mitochondrial Supplements on Mitochondrial Activity in Children with Autism Spectrum Disorder. J Clin Med. 2017, 6:18

Dhillon S, Hellings JA, Butler MG. Genetics and mitochondrial abnormalities in autism spectrum disorders: a review. Curr Genomics.2011, 12:322-332

Djordjevic D, et al. Two novel mitochondrial tRNA mutations, A7495G (tRNASer(UCN)) and C5577T (tRNATrp), are associated with seizures and cardiac dysfunction. Mitochondrion.2016, 31: 40-44

Dröse S, Brandt U, Wittig I. Mitochondrial respiratory chain complexes as sources and targets of thiolbased redox-regulation. Biochim Biophys Acta.2014, 1844(8): 1344-1354

Fillano JJ, Goldenthal MJ, Rhodes CH, Marín-García J. Mitochondrial dysfunction in patients with hypotonia, epilepsy, autism, and developmental delay: HEADD syndrome. J Child Neurol. 2002,17(6): 435439

Frye RE. Novel cytochrome B gene mutations causing mitochondrial disease in autism. J Pediatr Neurol.2012,10(01):035-040

Frye RE, Rossignol DA. Mitochondrial dysfunction can connect the diverse medical symptoms associated with autism spectrum disorders. Pediatr Res. 2011, 69(5Pt 2):41R-7R

Gargus JJ, Imtiaz F. Mitochondrial energy-deficient endophenotype in autism. Am J Biochem Biotech.2008,4:198-207

Giulivi C, et al. Mitochondrial dysfunction in autism. JAMA. 2010, 304(21): 2389-2396

Graf WD, et al. Autism associated with the mitochondrial DNA G8363A transfer RNA(Lys) mutation. J Child Neurol. 2000, 15: 357-361 
Greaves LC, et al. Mitochondrial DNA and disease. J Pathol. 2012, 226:274-286

Gu F, et al. Alterations in mitochondrial DNA copy number and the activities of electron transport chain complexes and pyruvate dehydrogenase in the frontal cortex from subjects with autism. Transl Psychiatry. 2013, 3(9): e299

Hadjixenofontos A, et al. Evaluating Mitochondrial DNA Variation in Autism Spectrum Disorders. Ann Hum Genet. 2013, 77(1): 9-21

Hoekstra JG, Hipp MJ, Montine TJ, Kennedy SR. Mitochondrial DNA mutations increase in early stage Alzheimer disease and are inconsistent with oxidative damage. Ann Neurol. 2016, 80: 301-306

lafrate AJ, et al. Detection of large-scale variation in the human genome. Nat Genet.2004, 36: 949-951

Kent L, Gallagher L, Elliott HR, Mowbray C, Chinnery PF. An investigation of mitochondrial haplogroups in autism. Am J Med Genet B Neuropsychiatr Genet. 2008, 147(6): 987-989

Koopman WJ, et al. Mammalian mitochondrial complex I: biogenesis, regulation, and reactive oxygen species generation. Antioxid Redox Sign.2010, 12(12):1431-1470

Koopman WJ, Willems PH, Smeitink JA. Monogenic mitochondrial disorders. N Engl J Med.2012, 366:1132-1141

Krysko DV, et al. Emerging role of damage-associated molecular patterns derived from mitochondria in inflammation. Trends Immunol.2011, 32(4):157-164

Legido A, Jethva R, Goldenthal MJ.Mitochondrial dysfunction in Autism. Semin Pediatr Neurol. 2013, 20:163-175

Lerman-Sagie T, Leshinsky-Silver E, Watemberg N, Lev D. Should autistic children be evaluated for mitochondrial disorders? J Child Neurol. 2004, 19: 379-381

Li H, et al. mtDNA heteroplasmy in monozygotic twins discordant for schizophrenia. Mol Neurobiol.2016, 54: 4343-4352

Li H, Slone J, Fei L, Huang T. Mitochondrial DNA Variants and Common Diseases: A Mathematical Model for the Diversity of Age-Related mtDNA Mutations. Cells.2019, 8(6):608

Lin MT, Beal MF. Mitochondrial dysfunction and oxidative stress in neurodegenerative diseases. Nature.2006, 443:787-795

Lombard J. Autism: a mitochondrial disorder? Med Hypotheses. 1998, 50: 497-500

Lord C, et al. Autism spectrum disorders. Neuron.2000, 28: 355-363 
Maenner MJ, et al. Prevalence of Autism Spectrum Disorder Among Children Aged 8 Years-Autism and Developmental Disabilities Monitoring Network, 11 Sites, United States, 2016. MMWR Surveill Summ.2020, 69(4):1-12

Malik AN, Czajka A. Is mitochondrial DNA content a potential biomarker of mitochondrial dysfunction? Mitochondrion. 2013, 13: 481-492

Napoli E, Wong S, Giulivi C. Evidence of reactive oxygen species-mediated damage to mitochondrial DNA in children with typical autism. Mol Autism.2013, 4(1): 2

Oka T, et al. Mitochondrial DNA that escapes from autophagy causes inflammation and heart failure. Nature.2012, 485(7397):251-255

Oliveira G, et al. Mitochondrial dysfunction in autism spectrum disorders: a population-based study. Dev Med Child Neurol. 2005,47(3):185-189

Palmieri L, Persico AM. Mitochondrial dysfunction in autism spectrum disorders: cause or effect? Biochim Biophys Acta.2010, 1797: 1130-1137

Patowary A, Nesbitt R, Archer M, Bernier R, Brkanac Z. Next Generation Sequencing Mitochondrial DNA Analysis in Autism Spectrum Disorder. Autism Res. 2017,10: 1338-1343

Persico AM, NapolioniV. Autism genetics. Behav Brain Res. 2013, 251:95-112

Pfeffer G, et al. New treatments for mitochondrial disease-no time to drop our standards. Nat Rev Neurol.2013, 9:474-481

Piryaei F, Houshmand M, Aryani O, Dadgar S, Soheili ZS. Investigation of the Mitochondrial ATPase 6/8 and tRNA(Lys) Genes Mutations in Autism. Cell. 2012, 14(2): 98

Pons R, et al.Mitochondrial DNA abnormalities and autistic spectrum disorders. J Pediatr. 2004,144: 8185

Redon R, et al. Global variation in copy number in the human genome. Nature. 2006, 444: 444-454

Risch N, et al. A genomic screen of autism: evidence for a mutinous etiology. Am J Hum Genet. 1999, 65: 493-507

Rose $S$, et al. Butyrate enhances mitochondrial function during oxidative stress in cell lines from boys with autism. Transl Psychiatry.2018, 8: 42

Rossignol DA, Frye RE. Mitochondrial dysfunction in autism spectrum disorders: a systematic review and meta-analysis. Mol Psychiatry.2012, 17: 290-314 
Rossignol DA, Rossignol LW, James SJ, Melnyk S, Mumper E. The effects of hyperbaric oxygen therapy on oxidative stress, inflammation, and symptoms in children with autism: an open-label pilot study. BMC Pediatr. 2007,7: 36

Schon EA, DiMauro S, Hirano M. Human mitochondrial DNA: roles of inherited and somatic mutations. Nat Rev Genet.2012,13(12):878-890

Schon EA, Przedborski S. Mitochondria: the next (neurode)generation. Neuron. 2011, 70:1033-1053

Shoffner $\mathrm{J}$, et al. Fever plus mitochondrial disease could be risk factors for autistic regression. J Child Neurol.2010, 25(4): 429-434

Tang G, et al. Mitochondrial abnormalities in temporal lobe of autistic brain. Neurobiol Dis.2013,54: 349361

Taurines R, et al. Expression analyses of the mitochondrial complex I 75-kDa subunit in early onset schizophrenia and autism spectrum disorder: increased levels as a potential biomarker for early onset schizophrenia. Eur Child Adolesc Psychiatry. 2010, 19(5): 441-448

Valenti D, de Bari L, De Filippis B, Henrion-Caude A, Vacca RA. Mitochondrial dysfunction as a central actor in intellectual disability-related diseases: an overview of Down syndrome, autism, Fragile X and Rett syndrome. Neurosci Biobehav Rev. 2014, 46: 202-217

Valiente-Pallejà A, et al. Genetic and clinical evidence of mitochondrial dysfunction in autism spectrum disorder and intellectual disability. Hum Mol Genet. 2018, 27(5): 891-900

Varga NÁ, et al. Mitochondrial dysfunction and autism: comprehensive genetic analyses of children with autism and mtDNA deletion. Behav Brain Funct.2018,14(1): 4

Wallace DC. A mitochondrial bioenergetic etiology of disease. J Clin Invest. 2013, 123(4): 1405-1412

Wallace DC. Mitochondrial DNA variation in human radiation and disease. Cell. 2015, 163: 33-8

Wallace DC, et al. Mitochondrial DNA mutation associated with Leber's hereditary optic neuropathy. Science.1988, 242(4884):1427-1430

Wang Y, Picard M, Gu Z. Genetic evidence for elevated pathogenicity of mitochondrial DNA heteroplasmy in autism spectrum disorder. PLoS Genet. 2016, 12(10): e1006391

Weissman JR, et al. Mitochondrial Disease in Autism Spectrum Disorder Patients: A Cohort Analysis. PLoS ONE. 2008, 3(11): e3815

Wong S, et al. Role of p53, Mitochondrial DNA Deletions, and Paternal Age in Autism: A Case-Control Study. Pediatrics.2016, 137(4): e20151888 
Ylikallio E, Suomalainen A. Mechanisms of mitochondrial diseases. Ann Med. 2012, 44:41-59

Yoo HJ, Park M, Kim SA. Difference in mitochondrial DNA copy number in peripheral blood cells between probands with autism spectrum disorders and their unaffected siblings. World J Biol Psychia.2017, 18(2): 151-156

Zhang Q, et al. Circulating mitochondrial DAMPs cause inflammatory responses to injury. Nature.2010, 464(7285): 104-107

Zsurka G, Kunz WS. Mitochondrial dysfunction and seizures: the neuronal energy crisis. Lancet Neurol.2015,14: 956-966

\section{Table}

Table 1 not available with this version

\section{Figures}

\section{Image not available with this version}

Figure 1 